\title{
Effects of discrimination training on stimulus generalization in pigeons: Role of resistance to extinction and response-produced stimuli
}

\author{
DAVID R. THOMAS, C. F. HICKIS, RAYMOND L. JACKSON, \\ and ROBERT J. NEWLIN \\ University of Colorado, Boulder, Colorado 80309
}

\begin{abstract}
In Experiment 1, two groups $(n=10)$ of pigeons received 17 sessions of TD (true discrimination) or ND (nondifferential) training with line angles. Seventeen sessions of SS (single stimulus) training with a wavelength preceded this training and two followed it. Subsequent wavelength generalization testing in extinction revealed a sharper TD than ND gradient. This slope difference was evident from the very first test stimulus presentation and remained stable throughout testing. As a consequence of substantial overtraining, there was no reduction of response strength and no sharpening of generalization during testing for either group. In Experiment 2, two groups $(n=16)$ of pigeons received 10 sessions of TD or PD (pseudodiscrimination) training with line angles, followed by four sessions of SS training with a single wavelength. During this training and in subsequent wavelength generalization testing in extinction, brief blackouts separated stimulus presentations. Again, the TD group yielded the sharper gradient. Although responding weakened and the gradients sharpened during the test, these effects were comparable in the two groups. Furthermore, gradients based on the percentage of trials with at least one response showed the same TD-PD slope difference. This finding indicates that differential control over responding by responseproduced feedback is inadequate to account for the TD-PD difference in generalization slope. Both experiments indicate that a purported difference in resistance to extinction is also an inadequate explanation.
\end{abstract}

Honig (1969) was the first to demonstrate that "extradimensional" discrimination training with one pair of stimuli may enhance the control gained subsequently by an entirely different stimulus. Honig trained one group of pigeons on a discrimination between different key colors (Group TD, for true discrimination). A second group (Group PD, for pseudodiscrimination) received the same sequence of stimuli, uncorrelated with reinforcement. Both groups were then reinforced for pecking at a set of vertical lines on the response key and finally received a generalization test, in extinction, along the dimension of line orientation. The TD group showed a significantly steeper angularity generalization gradient. Similar findings have been reported by many other investigators (e.g., Bresnahan, 1970; Mackintosh \& Honig, 1970; Thomas, Freeman, Svinicki, Burr, \& Lyons, 1970.)

This research was supported by research grants from NICHD (HD-03486) and NSF (BNS78-01407) to David R. Thomas. C. F. Hickis is now at Weber State College, Ogden, Utah. Requests for reprints should be addressed to: Dr. David R. Thomas, Department of Psychology, University of Colorado, Boulder, Colorado 80309.
Thomas et al. (1970) interpreted the TD-PD generalization slope difference as evidence for a process of "general attentiveness." They proposed that discrimination training in which one stimulus dimension is predictive of reinforcement will enhance attention to other stimulus dimensions which are not varied in training or are experienced later. Similarly, nondifferential training may teach subjects that the varied dimension is nonpredictive resulting in a lessened tendency to attend to other stimuli in the environment as well.

Mackintosh (1977) has recently criticized this interpretation on many grounds, one of which is a purported difference in resistance to extinction following TD and PD training. Friedman and Guttman (1965) and Thomas and Barker (1964) reported that wavelength generalization gradients obtained following single stimulus (SS) training became reliably sharper during the course of a generalization test in extinction. Mackintosh suggested that TD training reduces resistance to extinction relative to $\mathrm{PD}$ training, which presumably then results in greater sharpening of generalization slope during the course of testing for TD subjects. Even if it were shown that differences in the sharpening of generalization slope during ex- 
tinction contributed to the TD-PD generalization slope difference, the question would remain whether it is a necessary condition for obtaining that difference. In Experiment 1, we endeavored to determine whether a TD-PD difference comparable to that reported in the literature could be obtained when the amount of training was sufficient to insure no measurable extinction of responding during generalization testing, thus presumably no sharpening of gradients under either TD or PD conditions. Another change from the procedures used by Thomas et al. (1970) was the use of ND rather than a PD nondifferential training procedure. In PD training, the overall patterning of reinforced and nonreinforced periods is similar to that in TD; however, in TD, after the discrimination has been learned, most responding occurs during $\mathrm{S}+$ periods, thus reinforcement density while responding becomes greater under the TD condition. This does not occur, of course, if ND training is used, which matches the reinforcement density in the presence of both training stimuli to that present in S + for the TD group.

Our original purpose in using ND rather than PD training was particularly relevant to an "interdimensional" training experiment in which the test dimension was wavelength and one of the two training stimuli was also a wavelength value. In that situation, the $S+$ in TD training was reinforced on a variable interval 1-min (VI 1-min) schedule, whereas during PD training the schedule in the presence of that same wavelength value would have provided reinforcement half as often. Theoretically, this difference in reinforcement density could contribute to the flatter gradient in the PD group (cf. Hearst, Koresko, \& Poppen, 1964). To avoid this confounding, we used ND rather than PD training. In a direct comparison of generalization following ND and PD interdimensional training. Thomas and Wheatley (1974) determined that the effect of these two conditions was indistinguishable: both flattened generalization gradients by a comparable amount. This suggests that the effects of the nondifferential reinforcement contingency are so potent as to obscure any effect of reinforcement density.

In subsequent work in our laboratory, we have most often used ND rather than PD training in order to facilitate comparisons across inter- and extradimensional training paradigms. In a two-stage extradimensional procedure, such as that used by Honig (1969), ND and PD training should be interchangeable, since the stimulus of interest is not presented while these conditions are in effect.

As indicated earlier, substantial training was given in Experiment 1 in an effort to minimize extinction during the course of generalization testing (Hearst \& Koresko, 1968). In a two-stage experiment in which TD and ND training are first given with line angles and then SS wavelength training is adminis- tered, the more SS training given, the longer the interval between the initial training and the generalization test, thus the greater the opportunity for forgetting. This problem was circumvented and the likelihood of obtaining a large TD-ND difference maximized by combining the features of a proactive and a retroactive design in Experiment 1. Extensive wavelength training preceded the introduction of line-angle TD and ND training which was ultimately followed by two "reminder" sessions of additional SS wavelength training plus a brief wavelength "warm-up" immediately preceding the wavelength generalization test. Thus, despite the use of extended SS training, the TD or ND experience was quite "recent" at the time of generalization testing.

\section{EXPERIMENT 1}

\section{Method}

Subjects. The subjects were 20 experimentally naive adult domestic pigeons maintained at $75 \%$ of their free-feeding weights. The birds were housed in individual cages where access to grit and water was continuously available and a 16-h-light/8-h-dark cycle was in effect. Sessions occurred between $1 \mathrm{~h}$ and $12 \mathrm{~h}$ after lights on.

Apparatus. Four standard pigeon chambers were used (cf. Hickis, Robles, \& Thomas, 1977). Stimuli were projected onto the keys by Industrial Electronic Engineers in-line display cells (Series 0010) mounted $.64 \mathrm{~cm}$ behind the keys. Stimuli of nominal peak transmitted wavelengths $490,538,555,576$, and $606 \mathrm{~nm}$ were produced by Kodak Wratten filters $75,74,99,73$, and $72 \mathrm{~B}$, respectively. A white line, $3 \mathrm{~mm}$ wide $\times 21 \mathrm{~mm}$ long, in either the vertical or horizontal position was also used with an otherwise dark key. The filters were transilluminated by individual G.E. miniature lamps (No. 1815) drawing $200 \mathrm{~mA}$ current. The only other source of illumination in the chambers was provided by an ESB 24-V lamp situated above the food hopper and illuminated for the entire duration of each hopper presentation. Programming of scheduled events was accomplished by standard relay circuitry located in an adjoining control room.

Procedure. On the 1st day, all birds were magazine-trained, hand-shaped to peck the key, which was illuminated by a $555-\mathrm{nm}$ light, and allowed to earn 50 reinforcers delivered on a continuous reinforcement schedule. All subsequent training sessions were of $30 \mathrm{~min}$ duration. During the next 3 days, the mean interreinforcement interval was gradually lengthened until a VI 1-min schedule was in effect. Next, all subjects received 17 sessions of single stimulus training with the 555-nm light and a VI 1-min constant probability schedule with a minimum interreinforcement interval of $6 \mathrm{sec}$ and maximum of $160 \mathrm{sec}$. The subjects were then divided into two groups matched for response rate and received 17 sessions of TD or ND training, respectively. For the TD group, the vertical line signaled VI 1-min reinforcement; the horizontal line signaled extinction. The stimuli were randomly alternated for 60 -sec periods with no intervening blackouts, with the constraint that no more than three $\mathrm{S}+$ or three $\mathrm{S}$ - periods could occur consecutively. The ND group experienced the same patterning of horizontal and vertical line stimuli, except that the VI 1-min schedule was continuously in effect.

Following their line-angle training, all subjects received two "reminder" sessions of VI 1-min training with the 555-nm stimulus. On the test day, all subjects received a 10-min warm-up of VI 1-min reinforcement in the presence of $555 \mathrm{~nm}$ and then testing was begun. A series of blocks of five wavelengths (490, $538,555,576$, and $606 \mathrm{~nm}$ ) was successively presented with no responses being reinforced and no intervening blackouts. The stimuli remained on the key for $30 \mathrm{sec}$ each, and each block con- 
sisted of a different random ordering of each of the five stimuli. The same 12 different blocks of five chromatic stimuli were experienced in the same order by all birds in the experiment.

\section{Results and Discussion}

During the last session of discrimination training, the TD group responded a mean of 930.8 times to vertical and 135.2 times to horizontal, with a mean of $88.4 \%$ of total responses made to the vertical $(\mathrm{S}+)$ stimulus. Comparable figures for the ND group were 836.5 responses to vertical and 813.6 responses to horizontal, with a mean of $50 \%$ of total responses made to vertical.

Where resistance to extinction is to be compared following different experimental treatments, it is important that response strength be comparable at the conclusion of training. Performance during the two "reminder" sessions of SS training with the 555-nm stimulus just prior to testing indicated that the two groups were, indeed, comparable in response strength to this stimulus. Averaged across these two sessions, the TD group yielded a mean of 1,637 responses/ session compared to 1,674 responses in the ND group $(\mathrm{F}<1)$.

Next, it is appropriate to report the generalization gradients obtained from the TD and ND groups over the entire test session; this is the form in which such gradients have previously been reported. Figure la shows the absolute generalization gradients of the two groups, plotted in terms of mean total responses to the various test stimuli. Figure $1 b$ reports the relative gradients in terms of mean percent of total responses emitted to the different test values. These results are typical of those reported in the literature. In both the absolute and the relative comparisons, the TD gradient is sharper than the ND gradient. Because of large individual differences in levels of

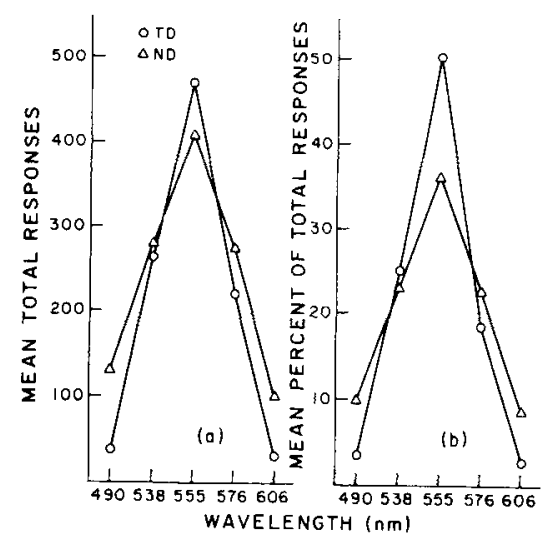

Figure 1. (a) Absolute generalization gradients (i.e., mean total responses) of TD and ND groups in Experiment 1. (b) Relative generalization gradients (i.e., mean percent of total responses) of TD and ND groups in Experiment 1. responding, however, the slopes of the absolute gradients are not significantly different; i.e., analysis of variance indicated that the interaction between group and stimuli was not reliable $[F(4,112)=1.74$, $\mathrm{p}>$.1]. The interaction was highly significant with the relative generalization measure, however $[F(4,112)$ $=3.64, \mathrm{p}<.01]$.

Having determined that TD and ND groups were responding comparably at the end of training and that the typical TD-ND generalization slope difference was obtained under the conditions used in this experiment, we may now seek evidence that differences in resistance to extinction were responsible for part or all of the generalization slope difference. To make this determination, the data presented in Figure 1 were reanalyzed in terms of successive quarters of the generalization test, i.e., blocks of three test series. In the analysis of the absolute gradients, there was no effect of blocks and no Blocks by Stimuli interaction (Fs $<1$ ). Mean responses in the four blocks were $245.3,273.4,260.9$, and 244.7 for the TD group and 321.4, 293.4, 310.0, and 268.5 for the ND group. Thus, there was no measurable extinction (i.e., weakening of responding) during generalization testing and no change in generalization slope. The analysis of relative generalization gradients indicated no interaction between groups, blocks, and stimuli $(F<1)$; i.e., the TD-ND difference in relative generalization slope did not change over the four blocks of testing.

The data reported above offer no support to Mackintosh's (1977) suggestion that following TD training more rapid extinction of responding, particularly to generalized test stimuli, contributed to the obtained difference in generalization slopes in TD and ND groups. It may be argued, however, that a more molecular analysis is required because extinction can occur even within the very first generalization test series, and its effects may generalize from one test stimulus to another. The critical test, therefore, involves responding to the first generalized stimulus experienced by the subject. To permit this comparison, the same test sequence had been administered to all subjects, with $606 \mathrm{~nm}$ the first test stimulus. The ND group responded a mean of 18.4 times during this stimulus exposure, compared to 9.2 times in the TD group. This difference is significant $[F(1,18)$ $=4.71, \mathrm{p}<.05]$, and it indicates a sharper TD than ND gradient prior to any opportunity for extinction to take place.

The results of Experiment 1 indicate that differential rates of extinction following TD and ND training are not necessary to produce the different generalization slopes typically associated with these training conditions. Experiment 2 was designed, in part, to ask whether differential rates of extinction contribute to the TD-ND generalization slope differ- 
ence obtained following typical (i.e., much briefer) training regimens. For this purpose, we used procedures similar to those employed by Honig (1969) in his original work on TD enhancement. For example, we used PD rather than ND training, and we trained the TD and PD birds for only 10 sessions, followed by only 4 sessions of SS training. Like Honig, we used brief blackouts separating stimulus presentations during both training and generalization testing. These blackouts permitted us to test another of Mackintosh's (1977) contentions, i.e., that the TD enhancement effect is due largely to the suppression of control by proprioceptive feedback from prior responding which normally masks control by the test stimuli following PD training. Tomie, Davitt, and Engberg (1976), using TD and PD autoshaping procedures, were able to find the TD enhancement effect despite long intertrial intervals without responding. However, their measure was still that of response rate during stimulus exposures, and thus was susceptible to influence by proprioceptive feedback. There is certainly less reason to expect that TD and PD training would result in differential proprioceptive control in autoshaping than in the free-operant paradigm. However, since the bulk of the relevant literature uses operant responding, it seemed most appropriate to test Mackintosh's assertion in that context. Where blackouts separate the presentation of generalization test stimuli, the first response to each test stimulus is presumably free of influence by prior responding and the latency of that response is thus an unbiased measure of associative strength. Thomas and Konick (1966) obtained systematic wavelength gradients following single stimulus training using this measure. If the measure of initial latency yields a sharper gradient following TD than following PD training, the proprioceptive feedback hypothesis will be shown to be inadequate to account for the TD enhancement effect.

In the Thomas and Konick (1966) study, no statistical comparisons were made between latency gradients of different groups, and the inherent variability of latency scores may preclude the finding of significant differences. Fortunately, other measures of generalized response strength are available which are also independent of prior responding. These include the number and percentage of presentations of the various test stimuli with at least one response. Because of our concerns about the variability of latency scores in Experiment 2, we ran larger groups of subjects $(n=16)$ than has been our typical practice.

\section{EXPERIMENT 2}

\section{Method}

Subjects. The subjects were 32 experimentally naive adult domestic pigeons maintained as in Experiment 1.
Apparatus. The same apparatus was used as in Experiment 1, with the exception that more resistance was used in series, with the bulbs transilluminating the 555- and 538-nm filters in order to partially correct for the greater transmittance of these filters.

Procedure. Initial training was similar to that used in Experiment 1 , with the following exceptions. The training stimulus was a white horizontal line on a dark surround, and there was only 1 day of lengthening the interreinforcement time after shaping. Subjects in the TD group received discrimination training in which the horizontal line signaled VI 1-min reinforcement and a vertical line signaled extinction. The stimuii were randomly alternated with the restriction that no more than three $\mathrm{S}+$ (horizontal line) or three $\mathrm{S}-$ (vertical line) periods could occur consecutively. All stimulus periods in training were $55 \mathrm{sec}$ in duration. They were separated by 5 -sec blackout periods, during which all chamber illumination was extinguished and reinforcement was unavailable.

Subjects in the PD group received the same sequence of the two training stimuli as the TD group, but during only half of the presentations of each stimulus, determined at random, was the VI 1-min schedule in effect. During the other half, reinforcement was unavailable. The TD and PD training were carried out for 10 daily 32 -min sessions.

On the next day, the birds were given the first of four sessions of SS training with a $555-\mathrm{nm}$ light on the key. On the test day, all subjects received a 10-min warm-up of VI 1-min reinforcement in the presence of $555 \mathrm{~nm}$ and then testing was begun. A series of blocks of five wavelengths $(490,538,555$, 576 , and $606 \mathrm{~nm}$ ) was successively presented; each block consisted of a different random ordering of each of the five stimuli. Test stimuli were of $25-\mathrm{sec}$ duration and were separated from each other by 5 -sec blackouts. There were eight different series of blocks used in this experiment, each used with two subjects from each group.

\section{Results and Discussion}

During the last session of discrimination training, the TD group responded a mean of 1,043.3 times to horizontal and 20.3 times to vertical, with a mean of $98.2 \%$ of total responses to the horizontal $(\mathrm{S}+)$ stimulus. Comparable figures for the PD group were 748.3 responses to horizontal and 726.4 responses to vertical, with a mean of $48.9 \%$ of total responses made to horizontal. Responding during blackouts was not recorded but was monitored auditorially, using relay clicks. By the end of discrimination training and throughout SS training and generalization testing, pecks during blackouts were rare and were invariably single pecks, early in the blackout, preceded by high-rate responding.

Averaged across the last two sessions of SS training with the 555-nm stimulus, the TD group yielded a mean of 1,864 responses/session, compared to 1,763 responses in the $P D$ group. These differences are not significant $(F<1)$ and thus provide a comparable baseline for the measurement of extinction during the course of generalization testing.

Next, it is appropriate to report the generalization gradients obtained from the two groups over the entire test session. Figure $2 a$ shows the absolute generalization gradients and Figure $2 \mathrm{~b}$ reports the relative ones. Both groups responded at comparable levels, the TD group giving a mean of 665 total responses, compared to 685 for the PD group $(F<1)$. In both plots, the TD gradient is sharper than that of the 


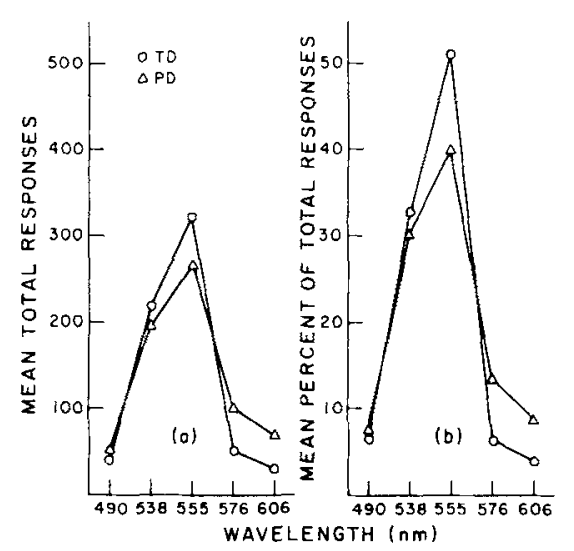

Figure 2. (a) Absolute generalization gradients of TD and PD groups in Experiment 2. (b) Relative generalization gradients of TD and PD groups in Experiment 2.

PD group. Analysis of variance of the absolute gradients of the TD and PD groups yielded an unreliable Group by Stimulus interaction $[F(4,120)=$ $1.58, \mathrm{p}>.10 \mathrm{l}$. In the analysis of the relative gradients, however, the Group by Stimulus interaction was significant $[\mathrm{F}(4,120)=3.53, \mathrm{p}<.01]$.

Having determined that TD and PD groups were responding comparably at the end of training and that the typical TD-PD generalization slope difference was obtained under the conditions of the present experiment, we may now seek evidence that differences in resistance to extinction were responsible for part or all of the generalization slope difference. Again, as in Experiment 1, the absolute generalization gradients were reanalyzed in terms of the four successive blocks of testing. The result indicated the expected weakening of responding over the course of the generalization test in extinction. Mean responses in the four blocks were $213.8,164.9,157.4$, and 126.7 for the TD group and 237.5, 193.4, 138.4, and 114.3 for the PD group. Analysis of variance indicates a significant effect of blocks $[F(3,90)=$ $11.84, p<.01$ ], but no effect of groups or Blocks by Groups interaction (Fs $<1$ ). However, the gradients do sharpen reliably, i.e., the Stimuli by Blocks interaction is significant $[\mathrm{F}(12,360)=2.20, \mathrm{p}<.02]$. The sharpening, however, is not differential; i.e., the Groups by Blocks by Stimuli interaction is insignificant $[F(12,360)=1.03, p>.1]$.

Friedman and Guttman (1965) and Thomas and Barker (1964) have pointed out that since responding extinguishes at different rates in different individuals, the most sensitive measure of changes in generalization during extinction is obtained when subjects are evaluated at comparable points in their extinction curves. To accomplish this, for each subject, the generalization test series were divided into four groups which represented as closely as possible the successive quarters of total responses. Relative generalization gradients based on these quarters are presented in Figure 3. It may be seen that the TD gradient is sharper than the PD gradient in each of the four quarters, and although the gradients become sharper during the course of testing, that sharpening is comparable in the two groups. Analysis of variance indicates a significant interaction between quarters and stimuli $[F(12,360)=5.53, p<.01]$, showing that the sharpening during testing is reliable. On the other hand, the $F$ for the interaction between groups, blocks, and stimuli is less than one.

We shall turn next to an analysis of the role of response-produced feedback in producing the TDPD generalization slope difference. In Figure 4 are plotted generalization gradients of the TD and PD groups, based on the latency of the first response during each stimulus presentation. Where no response was made, a latency of $25 \mathrm{sec}$ was recorded. As may be seen in the figure, the TD group produces a sharp latency gradient, with responding sub-

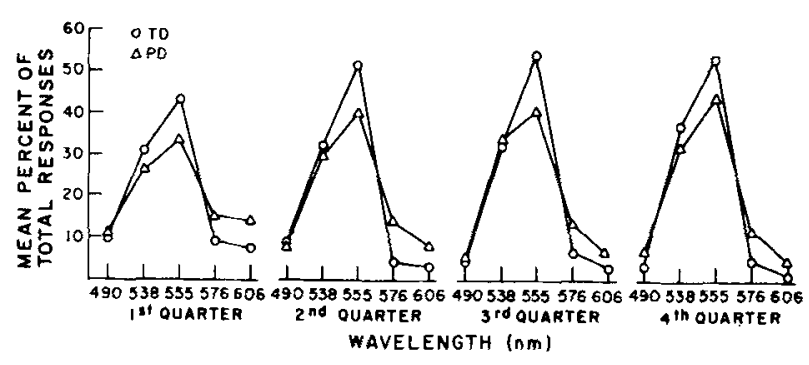

Figure 3. Relative generalization gradients of TD and PD groups over four quarters of testing in Experiment 2.

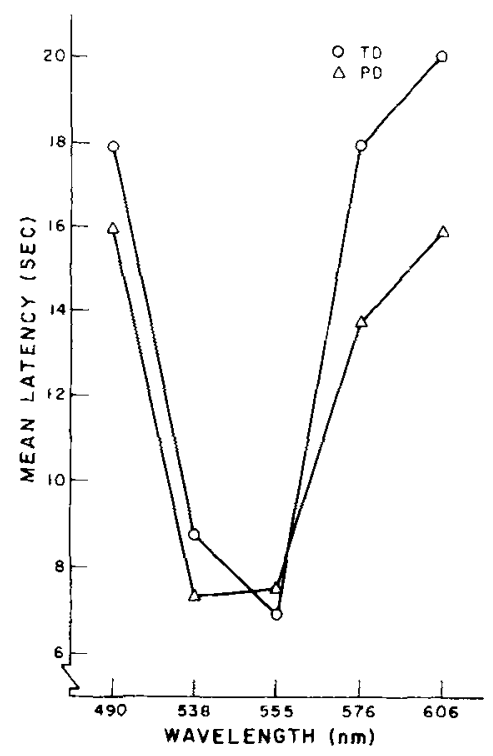

Figure 4. Generalization gradients of TD and PD groups based upon the latency of the first response on each test trial (stimulus
presentation) in Experiment 2. 
stantially more rapid to the training stimulus than to any other value. The gradient of the PD group is clearly flatter with responding equally rapid to 555 and $538 \mathrm{~nm}$. Nevertheless, the variance of the latency scores was such that analysis of variance failed to show a Group by Stimulus interaction $[F(4,120)=1.43, p>.1]$. A log transformation of the latency scores reduced variance somewhat but failed to produce significance. The relatively large mean latencies, especially to the stimuli farther from the training stimulus, indicate that the 5-sec blackout was sufficient to disrupt repetitive responding. The total latency between groups was not significantly different $[F(1,30)=1.96, p>.1]$.

The next measure considered was the number of stimulus presentations (trials) during which at least one response occurred. As Figure 5a indicates, sharp gradients are obtained with this measure and the gradient of the TD group is again sharper than that of the PD group. Again, however, the Group by Stimulus interaction does not reach significance $[F(4,120)=1.47, p>.1]$, and again there is no significant group effect $[F(1,30)=2.60, p>.1]$. The trials with a response gradient is, of course, an absolute gradient. Individual differences in rate of extinction and in level of responding, which generally prevent the obtaining of a reliable Group by Stimuli interaction with absolute total response gradients, as in both experiments reported here, have the same effect with the trials with a response measure. For each subject to contribute equally to the shape of the mean gradient, a relative gradient can be computed based on total trials with a response, analagous to relative gradients based on total responses. To obtain this measure, which is shown in Figure 5b, the proportion of trials with a response allocated to each of the five test stimuli was determined. Analysis of variance of these data yields a significant interac-

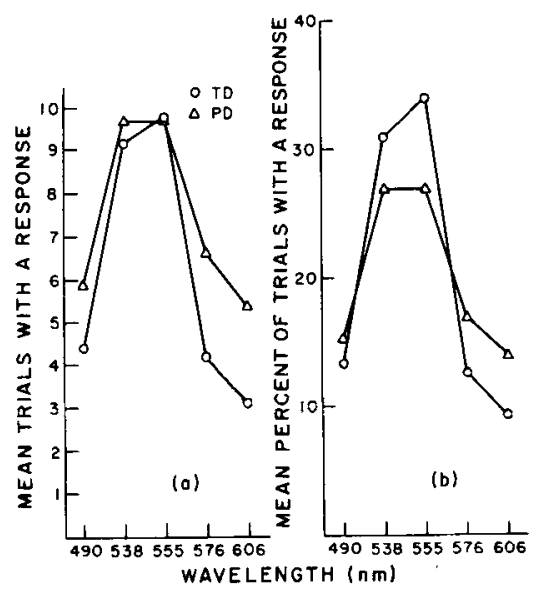

Figure 5. (a) Generalization gradients of TD and PD groups based upon the number of test trials with at least one response in Experiment 2. (b) Generalization gradients of TD and PD groups based upon the percent of all responded trials allocated to the various test stimuli in Experiment 2. tion between groups and stimuli $[F(4,120)=2.87$, $p<.03$ ]. Thus, with a measure of generalized responding that is independent of prior responding, the TD-PD difference in generalization slope is reliably obtained. Therefore, a difference in control over responding by response-produced stimuli is inadequate to account for the TD enhancement effect.

It was of interest to determine whether differential extinction in TD and PD groups accounted for part or all of the TD-PD generalization slope difference with the measure of proportion of responded trials. For this purpose, the data in Figure $5 \mathrm{~b}$ were reanalyzed in terms of each of the four successive blocks of generalization testing. The TD-PD difference in slope was present throughout testing, and although the gradients did sharpen throughout testing, they did not sharpen differentially.

\section{GENERAL DISCUSSION}

This study addressed two of several criticisms of the general attention position offered by Mackintosh (1977). The criticisms are very different in nature. The first is a purported difference in resistance to extinction following TD and PD training, which in turn purportedly results in greater sharpening of generalization during testing in extinction following TD training. In Experiment 1, we used overtraining, which produced no measurable extinction during testing, so we could ask whether differential sharpening of generalization was necessary to produce the TD-PD difference. It was not. In Experiment 2, we used a standard amount of training such that there was measurable extinction during generalization testing and measurable sharpening of the gradients. The sharpening, however, was comparable in the two groups. Thus, it may be concluded that the TDPD difference in generalization slope is not attributable to greater sharpening of TD gradients during the course of testing.

Mackintosh's (1977) second criticism of general attention is far more complex. In his review of the stimulus control literature, Mackintosh (1977) proposed the view that whenever TD training enhances control exhibited in generalization testing by an irrelevant stimulus, it does so by suppressing control by other (incidental) irrelevant stimuli which are present in PD training and may either overshadow the development of control by the irrelevant stimulus during training or may mask the demonstration of stimulus control by the irrelevant stimulus during generalization testing. Overshadowing is a learning deficit, whereas masking is a performance deficit. This distinction is vital to a conceptual analysis of experiments in stimulus control.

Mackintosh's masking-overshadowing interpretation of the TD-PD difference in generalization slope is consistent with the results of several investigators, including Hall and Honig (1974) and Turner and 
Mackintosh (1972). The Turner and Mackintosh study was the first to produce a retroactive TD enhancement effect. In their study, PD training with a vertical line superimposed on two different colors was followed by TD training with two different colors (without the line). The result was an angularity gradient as sharp as if the group had experienced TD training in Stage 1. Mackintosh argues (1977, p. 505), "That TD training with a new pair of stimuli can sharpen, for PD subjects, the gradient around a previously presented incidental stimulus implies that the typically flat gradient produced by PD subjects in free operant experiments cannot be a consequence of any failure of that incidental stimulus to acquire control. It must be due to a masking of such control by stimuli, which can then be neutralized by subsequent discrimination training." We shall argue later that the second statement does not follow inevitably from the first.

In the Hall and Honig (1974) study, Pavlovian TD training with differently colored houselights followed by autoshaping and operant keypeck SS training did not produce a TD enhancement effect. However, the interpolation of autoshaping, a TD procedure, makes these results difficult to interpret. (Although the 3 days of "autoshaping" were also inserted for keypeck-trained control groups, it is unlikely that the effect of this training was the same for these birds, which had previously had 17 days of keypeck training.)

Mackintosh (1977) also cites Wagner, Logan, Haberlandt, and Price (1968) for an example of a discrete-trial experiment which failed to produce the TD-PD effect. Presumably this is because proprioceptive feedback from prior responding plays no role in discrete-trial situations. However, Wagner et al. (1968) tested only one value along the incidental stimulus dimension. They found that TD subjects responded less to this value than did $P D$ subjects. They interpreted this as showing less control by that stimulus for TD than for PD subjects. In fact, the finding that TD subjects respond less is not at all incompatible with the concept of general attention. TD subjects should be more sensitive to all changes in stimuli and thus respond less than PD when only one stimulus from a compound is presented. General attention theory predicts that control within the dimension will also be greater for TD than for PD subjects. Thomas, Burr, and Eck (1970) replicated the Wagner et al. (1969) finding and showed that, despite a lower response rate, TD subjects allocated a greater proportion of the responses which they did make to the stimulus which was present during training, thus yielding a sharper relative gradient. Mackintosh (1977) criticized the Thomas et al. (1970) analysis on the grounds that a relative transformation might not be appropriate when the TD and PD response rates are so different. In experiments in which the TD and PD rates are comparable as a consequence of subsequent SS training, as in the present design, sharper TD gradients are also found. In the present experiments, the TD and PD gradients cross with both absolute and relative measures, and in all comparisons, so that there can be little doubt that the TD gradient is the sharper.

There are discrete-trial experiments other than that of Wagner et al. which show the TD enhancement effect. For example, Reinhold and Perkins (1955) found TD enhancement in a discrete-trial runway experiment using rats. The comparison groups in their experiment were two SS groups which either received consistent reinforcement or reinforcement matched to that of the TD group. Both SS groups produced flatter gradients along a constant irrelevant dimension than did the TD group. Note that one of the SS groups (that with consistent reinforcement) extinguished more rapidly than the TD group in that experiment as well. Tomie et al. (1976) demonstrated that a TD-PD difference could be obtained using a discrete-trial procedure (autoshaping), and Mullins and Winefield (1977) found the predicted positive transfer of TD vs. PD training to a new discrimination in a discrete-trial procedure (2- and 12-door choice situations). This pattern of findings, along with the results of our Experiment 2, indicates that differential control by feedback from prior responding is not necessary to produce the TD-PD difference.

The findings of a retroactive TD enhancement effect by Turner and Mackintosh (1972) and a parallel finding of a retroactive PD flattening effect by Honig (1974) have important implications for the general attention position, which we can only touch upon here. In its original conception, general attention was a learning construct; i.e., experience with TD training presumably led subjects to be more attentive to, and thus learn more about, subsequently experienced stimuli. We agree with Mackintosh that the retroactive studies indicate that $T D$ and $P D$ ef fects must be on performance rather than on learning. We disagree, of course, with his further conclusion that the effects must therefore be peripheral, i.e., a tradeoff between control by one class of incidental stimuli (those manipulated by the experimenter) and another (e.g., response-produced feedback). We prefer a central interpretation which might be put in signal detection terms (cf. Green \& Swets, 1974): TD training teaches the significance of stimuli, thereby tightening the decision criterion for responding to a novel stimulus as being the same as the training stimulus. Such an effect could be seen as readily in a retroactive design as in the more typical proactive one.

Blough (1969) has suggested that the slope of generalization gradients can be taken as an indication of the width of the response region between decision criteria. TD training, by arranging differential payoff (reinforcement) for two stimuli should lead to a narrowing of this region, whereas $P D$ and ND 
training, by arranging nondifferential payoff, should lead to its broadening. It is (perhaps) this tendency toward relatively narrow or broad response regions which is transferred to the new stimulus dimension, as reflected in differences in generalization or transfer performance. A signal detection analysis suggests a mechanism whereby general attentional effects may be mediated. It is not an alternative to the general attention position, but a possible refinement thereof. As such, it has the advantage of suggesting some new experimental manipulations which would otherwise not have appeared relevant.

It should be pointed out in closing that the results of our Experiment 2 rule out but one type of incidental stimulus as accounting for the TD-PD difference in generalization slope, i.e., response-produced feedback. The general tradeoff principle remains a tenable one. The problem is to specify the class of incidental stimuli purported to exert differential control following TD and PD training so that an empirical test can be performed. Rescorla and Wagner (1972) have called attention to contextual stimuli as an important source of control. Their theory would led to the expectation that control by contextual stimuli, e.g., houselight, white noise, etc., would be greater following PD than following TD training or SS training. This hypothesis was tested in part in a nonspecific transfer experiment reported by Newlin and Thomas (1978). Following either ND or SS training in Stage 1, the subjects were switched to a TD problem in Stage 2. For half of each group, the context condition (houselight off) was changed between the stages. This change led to improved TD performance in both groups, indicating that the context had indeed gained some control over responding, but the improvement (and therefore the amount of control by context) was not differential. In both context-change and non-context-change conditions, the ND group showed the same level of retardation. This finding suggests that a difference in contextual control is probably not responsible for the TD-PD difference in generalization slope, although a direct test should surely be made.

\section{REFERENCES}

Blough, D. S. Generalization gradient shape and summation in steady-state tests. Journal of the Experimental Analysis of Behavior, 1969, 12, 91-104.

Bresnahan, E. L. Effects of extradimensional pseudodiscrimination training upon stimulus control. Journal of Experimental Psychology, 1970, 85, 155-156.

Friedman, H., \& GutTman, N. Further analysis of the various effects of discrimination training on stimulus generalization gradients. In D. I. Mostofsky (Ed.), Stimulus generalization. Stanford: Stanford University Press, 1965.

Green, D. M., \& Swets, J. A. Signal detection theory and psychophysics. Huntington, N.Y: Krieger, 1974.

HALL, G., \& Honig, W. K. Stimulus control after extradimensional training in pigeons: A comparison of response contingent and noncontingent training procedures. Journal of Comparative and Physiological Psychology, 1974, 87, 945-952.

Hearst, E., \& Koresko, M. B. Stimulus generalization and amount of prior training on variable-interval reinforcement. Journal of Comparative and Physiological Psychology, 1968, 66, 133-138.

Hearst, E., Konesko, M. B., \& Poppen, R. Stimulus generalization and the response reinforcement contingency. Journal of the Experimental Analysis of Behavior, 1964, 7, 369-380.

Hickis, C. F., Robles, L., \& Thomas, D. R. Contextual stimuli and memory retrieval in pigeons. Animal Learning \& Behavior, $1977,5,161-168$.

Honig, W. K. Attentional factors governing the slope of the generalization gradient. In R. M. Gilbert \& N. S. Sutherland (Eds.), Animal discrimination learning. London: Academic Press, 1969.

HonIG, W. K. Effects of extradimensional discrimination training upon previously acquired stimulus control. Learning and $\mathrm{Mo}$ tivation, 1974, 5, 1-15.

Mackintosh, N. J. Stimulus control: Attentional factors. In W. K. Honig \& J. E. R. Staddon (Eds.), Handbook of operant behavior. Englewood Cliffs, N.J: Prentice-Hall, 1977.

Mackintosh, N. J., \& Honig, W. K. Blocking and attentional enhancement in pigeons. Journal of Comparative and Physiological Psychology, 1970, 73, 78-85.

Mullins, G. P., \& Winefield, A. H. Immunization and helplessness phenomena in the rat in a nonaversive situation. Animal Learning \& Behavior, 1977, 5, 281-284.

Newlin, R. J., \& Thomas, D. R. Nondifferential training retards acquisition of subsequent discriminations involving other stimuli. Animal Learning \& Behavior, 1978, 6, 385-390.

Reinhold, D. B., \& Perkins, C. C., JR. Stimulus generalization following different methods of training. Journal of Experimental Psychology, 1955, 49, 423-427.

Rescorla, R. A., \& Wagner, A. R. A theory of Pavlovian conditioning: Variations in the effectiveness of reinforcement and nonreinforcement. In A. H. Black \& W. F. Prokasy (Eds.), Classical conditioning II: Current research and theory. New York: Appleton-Century-Crofts, 1972.

Thomas, D. R, \& BARKer, E. G. The effects of extinction and "central tendency" on stimulus generalization in pigeons. Psychonomic Science, 1964, 1, 119-120.

Thomas, D. R., Burr, D. E. S., \& EcK, K. O. Stimulus selection in animal discrimination learning: An alternative interpretation. Journal of Experimental Psychology, 1970, 86, 53-62.

Thomas, D. R., Freeman, F., Svinicki, J. G., Burr, D. E. S., \& Lyons, J. The effects of extradimensional training on stimulus generalization. Journal of Experimental Psychology, 1970 , 83, Whole No. 1, Pt. 2, 1-21.

Thomas, D. R., \& Konick, D. S. A comparison of different measures of response strength in the study of stimulus generalization. Journal of the Experimental Analysis of Behavior, 1966, 9, 239-242.

Thomas, D. R., \& Wheatley, K. L. Effects of interdimensional training on stimulus generalization: An extension. Journal of Experimental Psychology, 1974, 103, 1080-1085.

Tomie, A., DavitT, G. A., \& Engberg, L. A. Stimulus generalization of autoshaped key-pecking following interdimensional and extradimensional training. Learning and Motivation, 1976, $7,240-253$

TURner, C., \& Mackintosh, N. J. Stimulus selection and irrelevant stimuli in discrimination learning in pigeons. Journal of Comparative and Physiological Psychology, 1972, 78, 1-19.

Wagner, A. R., Logan, F. A., Haberlandt, K., \& Price, T. Stimulus selection in animal discrimination learning. Journal of Experimental Psychology, 1968, 76, 171-180.

(Received for publication July 27, 1978; revision accepted November 22, 1978.) 